\title{
Veno-arterial extracorporeal membrane oxygenation as a bridge to cytolytic therapy
}

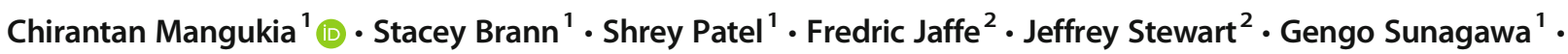 \\ Yoshiya Toyoda ${ }^{1}$
}

Received: 18 April 2020 /Revised: 15 June 2020 / Accepted: 19 June 2020 / Published online: 21 July 2020

(C) Indian Association of Cardiovascular-Thoracic Surgeons 2020

\begin{abstract}
We describe a case of successful use of veno-arterial (VA) extracorporeal membrane oxygenation (ECMO), as a bridge to cytoreductive therapy, in a patient with large mediastinal mass due to $\mathrm{T}$ cell lymphoblastic leukemia, complicated by acute cardiorespiratory compromise from mechanical compression and pulmonary embolism.
\end{abstract}

Keywords VA ECMO $\cdot$ Mediastinal neoplasms $\cdot$ Pulmonary embolism $\cdot$ Cytolytic therapy

\section{Introduction}

Large anterior mediastinal mass causing compression on vital structures like trachea, superior vena cava, pulmonary artery, right atrium, etc. is always challenging to manage. When indicated, surgical removal of such masses often requires mechanical circulatory support in the form of cardiopulmonary bypass or extracorporeal membrane oxygenation (ECMO). However, a large proportion of these tumors are lymphoma and leukemia, for which chemotherapy or cytolytic therapy is very effective. While undergoing or waiting for cytolytic therapy, these patients are at significant risk of sudden cardiorespiratory collapse because of critical compression of vital structures. We report a similar case here.

Electronic supplementary material The online version of this article (https://doi.org/10.1007/s12055-020-00992-3) contains supplementary material, which is available to authorized users.

Chirantan Mangukia

chirantan.mangukia@outlook.com

1 Division of Cardiovascular Surgery, Temple University Hospital, 3401 N. Broad Street, 3rd Floor Parkinson Pavilion, Philadelphia, PA 19140, USA

2 Department of Thoracic Medicine and Surgery, Temple University Hospital, Philadelphia, PA 19140, USA

\section{Case report}

A 21-year-old female was referred to our hospital with complaints of rapidly progressing shortness of breath and recent onset of dysphagia to solid food for the last 1 month. She had also noted progressive swelling of her face, hands, and lower extremities over the past few weeks. Her past medical history was significant for tonsillectomy and bronchial asthma, for which she was taking albuterol inhaler occasionally. She was an inconsistent smoker, not more than half a pack/day, for the last 2 years and was undergoing deaddiction treatment with nicotine patch. Computed tomography (CT) angiogram showed a large anterior mediastinal soft tissue mass causing rightward deviation and compression of the trachea and esophagus, partial collapse of left upper and lower lobes, and large left pleural effusion (Fig. 1a). The mass was also found to be causing compression on the main pulmonary artery and right ventricular outflow tract (Fig. 1b). CT also revealed extensive retrocrural and periaortic lymphadenopathy. Left thoracostomy tube drained a liter of straw-colored fluid. Cytology from pleural fluid showed abundant intermediate-sized lymphocytes, without Reed-Sternberg cells. CT-guided biopsy showed T cell lymphoblastic lymphoma. Baseline lactate dehydrogenase was 641 units/L with normal $\beta$-hCG levels.

Four hours after CT-guided biopsy, the patient had a syncopal attack followed by sudden increase in breathlessness. Electrocardiogram showed sinus tachycardia, and pulse oximetry showed profound hypoxia. She was immediately transferred to the Intensive Care Unit and was intubated. Despite intubation, ventilator optimization, and bronchodilators, she 
Fig. 1 a Large anterior mediastinal mass compressing pulmonary artery and right ventricular outflow tract (three white arrows). b Coronal view of the mass. $\mathbf{c}$ Right pulmonary artery thrombus (white arrow). d Left inferior pulmonary artery thrombus (white arrow)

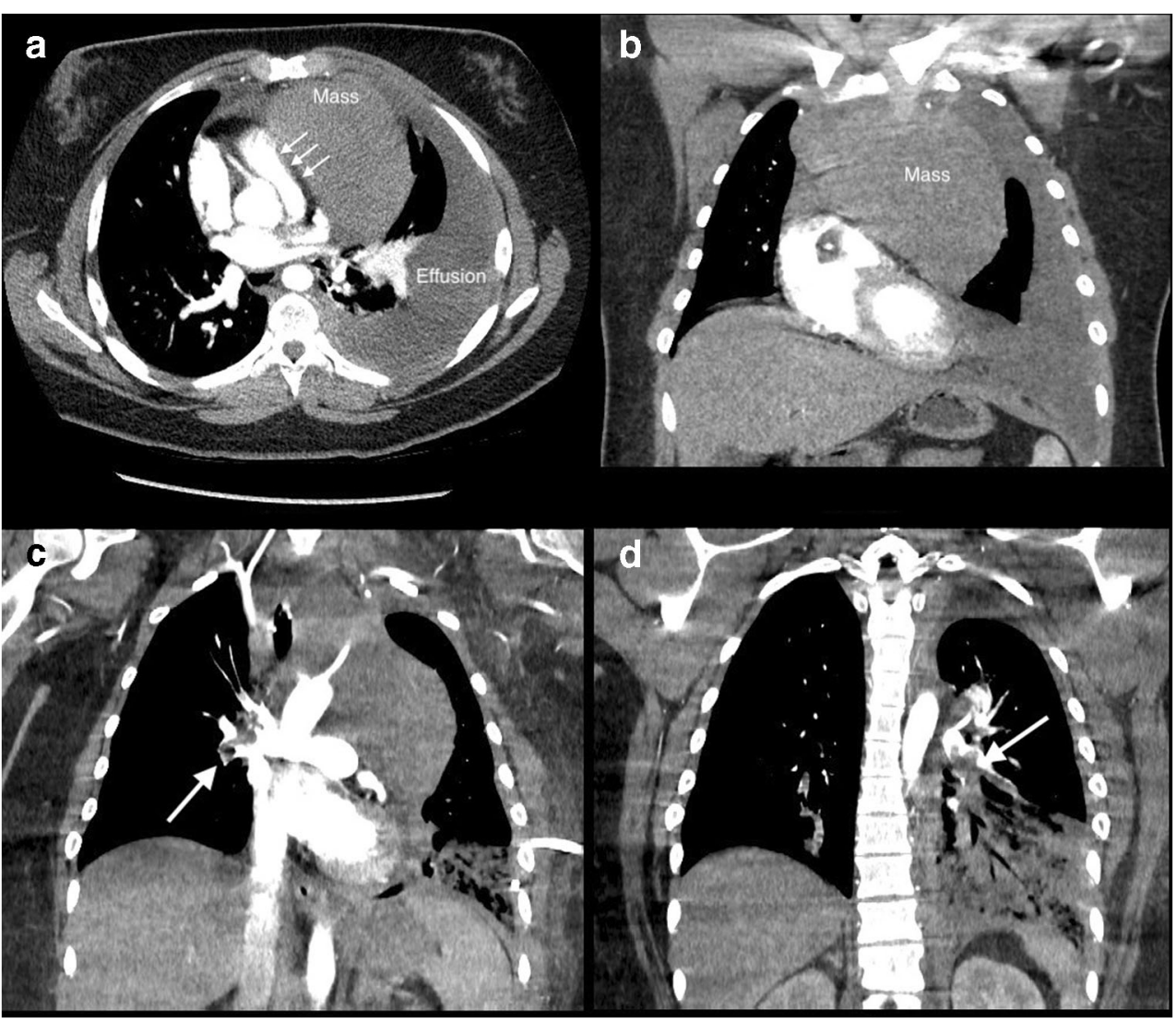

remained persistently hypoxic. Arterial blood gas showed metabolic acidosis (pH 7.29, pO2 $59 \mathrm{mmHg}$ and pCO2 $38 \mathrm{mmHg}$, base deficit of 8) with $100 \% \mathrm{FiO} 2$ on ventilator with optimum pressure-volume settings (tidal volume of $480 \mathrm{ml}$ and positive end expiratory pressure of $10 \mathrm{~cm}$ of water) and complete muscle paralysis. At this point, only minimal dose of vasopressin was required to support mean arterial blood pressure (which was $75 \mathrm{mmHg}$ ), but extremities were cold, and arterial blood gas suggested high possibility of low cardiac output. On clinical examination, central venous pressure was very high and was estimated to be above $15 \mathrm{~cm}$ of water.

Two major differential diagnoses were tabled: (1) acute submassive pulmonary embolus (PE) and (2) post-biopsy hemorrhage inside the tumor leading to sudden enlargement of mass and compression on the heart. A quick bedside screening transthoracic echocardiography (TTE) showed normal sized left and right ventricle $(\mathrm{RV})$ with good contractility but with partial RV outflow tract obstruction. A large mass was clearly visible anterior to the pericardium. Bedside emergent chest X-Ray did not show any significant increase in the mass size. She was considered unstable for emergency CT scan.

Since she only needed minimal vasopressin and screening echocardiography looked relatively unremarkable, discussion took place for choice of ECMO - veno-venous vs veno-arterial. Potential cardiac compression (as reflected by high jugular pressure), suspicion of sub-massive PE, cold extremities, clinical suspicion of low cardiac output, and worsening metabolic acidosis were major determining factors behind our decision of emergent VA ECMO. Peripheral VA ECMO comes with a caveat of limb ischemia risk, but she was young and had no history of peripheral vascular disease. Therefore, VA ECMO was established in percutaneous fashion using groin vessels. A $6 \mathrm{~F}$ distal perfusion catheter was placed and connected to ECMO circuit to avoid limb ischemia.

After stabilization, detailed bedside TTE showed large mass anterior to pericardium causing significant compression of RV and RV outflow tract, albeit on ECMO with fully drained RV (Videos 1 and 2). After initiation of ECMO, arterial blood gas showed $\mathrm{pH} 7.34, \mathrm{pO} 2107 \mathrm{mmHg}, \mathrm{pCO} 2$ $37 \mathrm{mmHg}$, and base deficit of 5 on ECMO flow of $4.8 \mathrm{~L}$ and sweep gas of $4 \mathrm{~L}$.

$\mathrm{CT}$ angiogram showed new finding of right pulmonary artery and left lower lobar artery thrombus (Fig. 1c, d) and ruled out hemorrhage in the mass. Intravenous heparin infusion was started and titrated with target activated prothrombin time of 60-70 s. Retrospectively thinking, compression caused by mass prevented RV dilation and caused tamponade physiology, completely masking the usual echocardiographic picture of acute PE.

Dexamethasone as a cytoreductive agent along with allopurinol and rasburicase, to prevent tumor lysis syndrome, was started as per hematology recommendation. In a 
multidisciplinary meeting, it was decided that surgical debulking was inappropriate from hematological point of view and was reserved as a last resort. So it was decided to use VA ECMO as a bridge to interventional suction thrombectomy and cytoreductive therapy.

Next day, she underwent a successful suction thrombectomy in intervention radiology suit using a FlowTriever device (Inari Medical) through right internal jugular vein, under ECMO support.

Within a day after initiation of the cytoreductive therapy, lactate dehydrogenase level jumped beyond 1000 units/L within $24 \mathrm{~h}$ suggesting rapid tumor cell lysis. Two days after initiation of cytoreductive therapy, bedside TTE showed significant hemodynamic relief with reduction in mass size sitting anterior to pericardium (Video 3). ECMO weaning ramp study was found to be encouraging. Next day, after total 3 days of uncomplicated ECMO support, she was successfully weaned off ECMO and decannulated in the operating room with direct surgical repair of femoral artery. Further cytoreductive chemotherapy was well tolerated during the hospital course. Two days after decannulation, she was transferred to another hospital, specialized in hematological malignancies and chemotherapy, with minimal ventilator support.

\section{Discussion}

The need for emergency ECMO for cardiorespiratory compromise in mediastinal malignancy is uncommon. There are few case reports in the literature which describe airway and vena caval compression by mediastinal mass requiring ECMO [1-3]. Our case report is a unique combination of large mediastinal mass with pulmonary artery and RV outflow tract compression complicated by acute sub-massive pulmonary embolism causing cardiorespiratory collapse. PubMed search using MESH words "Mediastinal Neoplasms" AND "Extracorporeal Membrane Oxygenation" AND "Pulmonary Embolism" showed 0 results. Scopus and Google Scholar search using the same words did not reveal any report similar to ours.

Given the screening echocardiography findings, we did not hesitate to proceed with VA ECMO considering potential compression, sub-massive PE suspicion, and metabolic acidosis. In otherwise healthy young individuals without any underlying cardiac disease or sepsis, sinking blood pressure is usually the last sign of low cardiac output. It is very important to identify low cardiac output earlier in the clinical course and treat appropriately, in our case using ECMO, to prevent end organ ischemia.

In conclusion, presence of large anterior mediastinal mass may mask echocardiographic signs of acute PE, and critical decisions must rely on clinical judgment. VA ECMO can be used as a bridge to cytoreductive therapy in presence of hemodynamic compromise.

Funding The authors do not have any pertinent funding disclosures to describe.

\section{Compliance with ethical standards}

Conflict of interest The authors declare that they have no conflict of interest.

Research involving human participants and/or animals Not applicable as per institutional ethical committee as the scientific information presented in paper does not fall into category of clinical trial or usage of experimental modalities.

Informed consent Obtained.

\section{References}

1. Balasubramanian S. Successful extracorporeal CPR of a patient with a bulky mediastinal mass and acute airway compromise. Chest. 2019;156:A1859-60.

2. Carro SE, Essex DW, Alsammak M, Bains A, Toyoda Y, Keshavamurthy S. Mediastinal lymphoma presenting in cardiogenic shock with superior vena cava syndrome in a primigravida at full term: salvage resection after prolonged extracorporeal life support. Case Rep Oncol. 2019;12:401-10.

3. Ueda Y, Hirayama I, Horie R, Doi K, Morimura N. Central airway obstruction due to mediastinal malignant lymphoma requiring venovenous-extracorporeal membrane oxygenation. Case Rep Acute Med. 2019;2:42-7.

Publisher's note Springer Nature remains neutral with regard to jurisdictional claims in published maps and institutional affiliations. 